\title{
Obituary
}

CARDIOLOGY

Published online: April 3, 2018

DOI: $10.1159 / 000488037$

\section{Raymond John Lipicky, MD}

Raymond John Lipicky, a member of our Editorial Board for almost 15 years, died on February 16 at the age of 85. At the time of his death, he was Director of LIPICKY, LLC. (established to consult to industry about the development of therapeutics). However, Ray Lipicky was far better known for his 21 years of service to the nation as Director, Division of Cardio-Renal Drug Products, Office of Drug Evaluation I, Center for Drug Evaluation and Research of the US FDA. In that position, he built on the work of Robert Temple, who had preceded him and had moved to broader FDA responsibilities, in bringing critical scientific analysis to considerations of approval of cardiovascular and renal drugs. Previously, he spent 14 years at the University of Cincinnati College of Medicine (his alma mater, where he had also trained in cardiology) as Professor of Pharmacology, Professor of Medicine, and Director, Division of Clinical Pharmacology. He was also a Visiting Scientist at the Marine Biological Laboratory (Woods Hole, MA, USA), where, for about 30 years, he spent his summers pursuing an interest in drug effects on electrically excitable membranes, experimenting in squid axons.

Ray influenced and taught several generations of clinical trialists and other cardiological scientists by his incisive insights and critiques of FDA New Drug Applications and by his lectures and discussions at medical meetings. He also trained and served as a model for his successors as Director of the Cardio-Renal Division, including the current superb Director, Norman Stockbridge. His teaching efforts were many, but notably included his annual contributions for 23 years to "Advanc- es in Cardiovascular Pharmacology and Devices: Protocol Design and Methodology. The U.S. Regulatory Process" [1] (sponsored by the American Institute of Therapeutics and directed by John Somberg, MD, an Associate Editor of this journal) and as a co-director and annual contributor to the annual Cardiovascular Clinical Trialists Forum and Workshop, now in its 15 th year and overseen by Faiez Zannad.

I first met Ray in 1981, his first year with the FDA, when he was assigned as FDA reviewer for an antihypertensive drug presented to the FDA Cardio-Renal Drugs Advisory Committee, of which I was in my first year as Chairman. The drug had failed to gain FDA approval on previous hearings and its sponsor had abandoned the drug, believing that a fair hearing was not possible. Ray's critique was positive and was so compelling that the Committee voted unanimously to recommend approval. The drug was never marketed but scientific integrity and fairness were established. Ray's primary principle was that the data, rather than theory, must drive therapeutics evaluation. His well-reported dictum that, if swamp water were presented to the FDA for approval and it reduced symptoms and mortality without unacceptable adverse effects for its indicated use, he would approve it with no knowledge of its "mechanism of action." This became a rock-solid principle of FDA cardiovascular drug evaluation.

For several years, this consideration clearly separated the FDA from some non-US regulatory agencies that tended to rely heavily on putative "mechanisms of action." The flaws of the latter approach were illustrated 
most recently for cyclo-oxygenase 2-selective (COX-2-selective) non-steroidalanti-inflammatorydrugs(NSAIDs). When data began to emerge that one of these (rofecoxib) increased major adverse cardiac events, including mortality (albeit largely based on data with doses higher than those for which it was approved in the USA), the manufacturer withdrew the popular drug from the market.

In 2005, the FDA (from which Ray had already retired) held a hearing to assess the fitness of all approved NSAIDs and, specifically, the relative safety of COX-2-selective versus -non-selective (ns) NSAIDs. A comprehensive review of the data at an open meeting led the FDA to conclude a lack of rigorous evidence of cardiovascular risk greater than benefit and, specifically, little evidence of differential relative risk among ns-NSAIDs and COX-2 selectives. The FDA added a statement about the possible cardiovascular risk of all NSAIDs to the packaging labels (instructions for use [IFU]). However, the FDA concluded that definitive data were needed for such widely used drugs and mandated the performance of a relative safety study of the single remaining US-approved COX-2-selective NSAID, celecoxib, and popular ns-NSAIDs, ibuprofen and naproxen.

Working from the same data, the European Medicines Agency concluded that COX-2 selectives as a group were particularly dangerous for patients with coronary artery disease and contraindicated such drugs from use in these patients in Europe. There followed the 25,000-patient PRECISION [2] trial, which took 10 years to complete (by which time the manufacturer's patent on celecoxib had expired). PRECISION found that the FDA had been cor- rect, showing no excessive cardiovascular adversity with celecoxib compared with the 2 ns-NSAIDs (and less gastrointestinal or renal adversity than with the comparators). PRECISION was long, arduous, and expensive, but it served to prove one of the principles on which Ray's professional life was founded: data must drive the evaluation process.

I never spoke with him about his views of PRECISION in which I was involved as a protocol developer and member of the Executive Committee. I am sure he would have found many flaws in the study and the process. However, a fitting epitaph for Ray is that the American people benefitted enormously from his scientifically critical nature and his Socratic method of questioning scientific dogma. His contributions to cardiovascular drug development were many, but his analytical, questioning approach to data evaluation will constitute his greatest legacy to the scientific community.

Jeffrey S. Borer, MD, New York, NY, USA

\section{References}

1 Borer JS, Somberg JC (eds): Cardiovascular Drug Development: Protocol Design and Methodology. New York, Marcel Dekker, Inc., 1999.

2 Nissen SE, Yeomans ND, Solomon DH, Lüscher TF, Libby P, Husni ME, Graham DY, Borer JS, Wisniewski LM, Wolski KE, Wang Q, Menon V, Gaffney M, Beckerman B, Berger MF, Bao W, Lincoff AM; PRECISION Trial Investigators: Cardiovascular safety of non-steroidal anti-inflammatory drugs in patients with chronic arthritis. N Engl J Med 2016;375: 2519-2529. 